\title{
Ibio-Number Assay: A Physicochemical Assay that Predicts the Bioactivity of Erythropoietin with High Precision and Accuracy and May Replace the Mouse Bioassay in the Quality Control of EPO Batch Release
} Hermentin $\mathbf{P}^{*}$

Feuerdornweg 8, 35041 Marburg, Germany

\begin{abstract}
The Ibio-number assay, based on capillary zone electrophoresis data of EPO samples, is evaluated as a physicochemical assay that enables to calculate the bioactivity of EPO medicinal products.

In part 1, the CZE data of the candidate biological reference preparation EPO CBRP3 of a collaborative study of 10 laboratories (published in 2007) are used to calculate the bioactivity of EPO CBRP3 and to determine the interlaboratory precision of the assay and its accuracy against the stated bioactivity of EPO BRP3. This retrospective analysis by the author revealed an inter-laboratory precision of $\mathrm{CV}=0.8 \%$ ( $\mathrm{n}=9$ labs, 1 outlier lab excluded). The bioactivity calculated for cBRP3 $(139.6 \mathrm{IU} / \mu \mathrm{g})$, compared with the stated bioactivity of BRP3 $(141.1 \mathrm{IU} / \mu \mathrm{g}=100 \%)$, provided an accuracy of $98.9 \%$.

In part 2, the CZE data of an epoetin alfa drug substance secondary standard and two epoetin alfa drug product samples $(40,000 \mathrm{IU} / \mathrm{mL}$ and $2,000 \mathrm{IU} / \mathrm{mL})$ from Centocor and two artificial epoetin beta concentrated solution samples from Roche (the mean of 40 epoetin beta 'training batches' and the mean of 17 epoetin beta 'validation batches') were retrospectively analyzed by the author using the Ibio-number assay. Moreover, the two hypothetical epoetin alfa/beta 1:1 mixtures of the Centocor and the Roche concentrated solution materials were prepared and the Ibionumber compared with the stated bioactivity of EPO BRP3. The results are summarized as follows: The lbio-number assay applied to Centocor's epoetin alfa secondary standard provided a potency of $142.3 \mathrm{lU} / \mathrm{\mu g}$. The same assay applied to the mean of the 40 epoetin beta 'training batches' respectively the mean of the 17 epoetin beta 'validation batches' from Roche provided potencies of $133.8 \mathrm{IU} / \mathrm{\mu g}$ and $139.9 \mathrm{IU} / \mathrm{\mu g}$, respectively. The two hypothetical alfa/beta $1: 1$ mixtures yielded bioactivities of $138.0 \mathrm{IU} / \mathrm{\mu g}$ and $141.1 \mathrm{IU} / \mathrm{\mu g}$, respectively, which matched the stated bioactivity of BRP3 $(141.1 \mathrm{IU} / \mathrm{\mu g})$ with accuracies of $97.8 \%$ and $100.0 \%$, respectively.
\end{abstract}

The Ibio-number assay applied to Centocor's formulated 40,000 IU/mL and 2,000 IU/mL epoetin alfa drug product samples revealed that the polysorbate 80 removal from the formulated samples decreased the bioactivity of the products by $-1.7 \%$ ( $40 \mathrm{k}$ sample) respectively $-2.9 \%$ ( $2 \mathrm{k}$ sample).

This data demonstrates the suitability of the Ibio-number assay to calculate and compare the bioactivity of EPO samples with up till now unmet precision and accuracy.

It is suggested that a prospective collaborative validation study will show that the lbio-number assay is suitable to replace the mouse bioassay of EPO drug substance batch release, which will provide an increase in bioactivity precision and accuracy without any loss of product safety.

Running Title: Ibio-Number and EPO Bioactivity

Keywords: Bioassay; Biological reference preparation (BRP); Capillary zone electrophoresis (CZE); Epoetin; Potency; Standard

Abbreviations: BNM: Bioassay in Normocythaemic Mice; BRP: Biological Reference Preparation; cBRP: Candidate BRP; CV: Coefficient of Variation; CZE: Capillary Zone Electrophoresis; EPO: Erythropoietin; HPAEC:High-Performance Anion-Exchange Chromatography; F-EPO: Formulated Erythropoietin; Ibio-number: Isoform Number Calculated via the Bioactivities of the Isoforms Separated in CZE; IF: Isoform; in: Individual Isoform Number Shares; IU: International Unit; MV: Mean Value; Ph. Eur.: European Pharmacopoeia; pn: Peak Area Percent Shares; RP-HPLC: Reversed-Phase High-Performance Liquid Chromatography

\section{Introduction}

In recent years, there have been efforts to replace the highly contested (consumption of animals) and highly inaccurate $(\mathrm{CV} \approx 25 \%$ $[1,2], \approx 20 \%$ [3]; uncertainty $15-30 \%$ [4]; as stated by Zimmermann et al. [3]) polycythaemic and normocythaemic mouse bioassays in the quality control of erythropoietin [5] by more precise and more accurate physicochemical methods such as Z-number determination based on ion-exchange chromatography of the isolated N-glycans (glycan mapping) [2,6,7] or I-number determination [8] based on the native protein and the peak areas of the various isoforms separated in CZE $[9,10]$. This approach has recently been supported by a study from Roche [3] that aimed at replacing the normocythaemic mouse bioassay for epoetin beta batch release on the basis of primarily CZE data. In contrast to the I-number assay which uses all eight EPO isoforms separated in CZE (where applicable), the Roche assay uses only five isoforms and adds instead a term reflecting sialylation. In

*Corresponding author: Hermentin P, Feuerdornweg 8, 35041 Marburg, Germany Ph: +49 6420 82090; E-mail: peter.hermentin@t-online.de

Received January 17, 2017; Accepted February 03, 2017; Published February 06, 2017

Citation: Hermentin P (2017) Ibio-Number Assay: A Physicochemical Assay that Predicts the Bioactivity of Erythropoietin with High Precision and Accuracy and May Replace the Mouse Bioassay in the Quality Control of EPO Batch Release. Pharm Anal Acta 8: 533. doi: 10.4172/2153-2435.1000533

Copyright: $\odot 2017$ Hermentin P. This is an open-access article distributed unde the terms of the Creative Commons Attribution License, which permits unrestricted use, distribution, and reproduction in any medium, provided the original author and source are credited. 
Citation: Hermentin P (2017) Ibio-Number Assay: A Physicochemical Assay that Predicts the Bioactivity of Erythropoietin with High Precision and Accuracy and May Replace the Mouse Bioassay in the Quality Control of EPO Batch Release. Pharm Anal Acta 8: 533. doi: 10.4172/21532435.1000533

Page 2 of 9

the meanwhile, Roche's physicochemical assay has been accepted for epoetin beta drug substance batch release in Europe [11].

The aim of this retrospective analysis by the author of the CZE data of

i. $\quad$ EPO cBRP3 of a collaborative study of 10 laboratories published in 2007 [12]

ii. An epoetin alfa drug substance secondary standard from Centocor [13]

iii. Two artificial epoetin beta drug substance batches from Roche, hypothetically constructed from 40 epoetin beta 'training batches' and 17 epoetin beta 'validation batches' [3]

iv. Two hypothetical epoetin alfa/beta 1:1 mixtures

was to show that the Ibio-number assay is suitable to assess the bioactivity of EPO samples with high precision and accuracy and may thus be a candidate physicochemical assay to replace the mouse bioassay in the quality control of EPO batch release.

\section{Materials and Methods}

\section{Materials}

EPO cBRP3 was as described in a collaborative study published in 2007 [12], and the CZE data were taken from this study. EPO cBRP3 consisted in "a 50:50 (weight/weight) blending of 2 erythropoietin preparations corresponding to products currently available on the European market (erythropoietin-alfa and erythropoietin-beta) with "a potency of 35,280 IU/vial " [12], i.e., 141,120 IU/mg or $141.1 \mathrm{IU} / \mu \mathrm{g}$, used throughout herein.

Epoetin alfa secondary standard was from Centocor as described elsewhere [13], and the CZE data were taken from this study. Briefly, the epoetin alfa sample from Centocor consisted in epoetin alfa obtained from Johnson \& Johnson Ortho Biotec and was used to prepare a secondary standard that has been calibrated against EPO BRP1 [13].

Epoetin alfa drug product samples formulated with polysorbate 80 to contain $2,000(2 \mathrm{k})$ and $40,000(40 \mathrm{k})$ International Units, equivalent to $16.8 \mu \mathrm{g} / \mathrm{mL}$ and $336.0 \mu \mathrm{g} / \mathrm{mL}$ epoetin alfa, respectively, were likewise as described [13]. The CZE data of these drug product samples measured after polysorbate 80 removals were again taken from this Centocor study.

Two artificial epoetin beta materials were hypothetically constructed from epoetin beta concentrated solution batches from Roche, "derived from several variants of a well-defined manufacturing procedure which is tightly controlled within defined limits" [3]: i) A pool of 40 epoetin beta 'training batches' and ii) A pool of 17 epoetin beta 'validation batches'. The CZE data published by Roche were those of the 40 epoetin beta training batches. Noteworthy, in these 40 epoetin beta training batches "batches were also included with markedly low biological potencies" [3], whereas the specific activities of the 17 validation batches, determined in normocythaemic mice, were of the medium range of Roche's well established NeoRecormon ${ }^{\circ}$ production.

Two hypothetical epoetin alfa/beta 1:1 mixtures were constructed from Centocor's epoetin alfa secondary standard [13] and i) The mean of Roche's 40 epoetin beta training batches respectively ii) The mean of Roche's 17 epoetin beta validation batches [3].

\section{Methods}

The Ibio-numbers were calculated as earlier described, however the Ibio-number has then not been termed as such $[9,10]$. Thus, the overall Ibio-number of an EPO sample is the sum of the products of the individual CZE peak area percent shares (pn) and isoform factors Fi corresponding with the respective isoform bioactivities $[9,10]$ (Formula 1):

$\mathrm{Ibio}=\mathrm{p} 1 \times \mathrm{F} 1+\mathrm{p} 2 \times \mathrm{F} 2+\mathrm{p} 3 \times \mathrm{F} 3+\mathrm{p} 4 \times \mathrm{F} 4+\mathrm{p} 5 \times \mathrm{F} 5+\mathrm{p} 6 \times \mathrm{F} 6+$ $\mathrm{p} 7 \times \mathrm{F} 7+\mathrm{p} 8 \times \mathrm{F} 8$

(Formula 1)

For epoetin alfa and epoetin beta, the factors Fi were derived from the bioactivities of the individual isoforms as published by Amgen for epoetin alfa [14] (providing Ibio_A) (Table 1a) respectively Roche for epoetin beta [15] (providing Ibio_R) (Table 1b). As the isoform bioactivities were published as "units/mg erythropoietin polypetide" [14] respectively "IU/mg protein" [15], the 'primary' Ibio-numbers were "multiplied by the factor 0.60 polypetide/mg erythropoietin glycoprotein to give specific activities expressed as units $/ \mathrm{mg}$ erythropoietin glycoprotein" [14] respectively the 'final' Ibio-numbers.

Isoforms 1-8 (where applicable) were used for Ibio-number calculation.

The Ibio-number of cBRP3 (Ibio_cBRP3) was retrospectively calculated by the author according to Formula 1 from the CZE data given in the collaborative study published in 2007 [12]. The final Ibionumber for cBRP3 was the arithmetic mean of Ibio_A and Ibio_R (Table 1). This final Ibio-number ("mean of means") of cBRP3 was used to assess the inter-laboratory precision of the assay ( $n=9$ labs) and the accuracy against the stated bioactivity of BRP3 (141.1 IU/ $\mu \mathrm{g})$ set $100 \%$.

Note: Outliers according to Grubbs [16] respectively Dixon [17], as retrospectively identified by the author in the collaborative study [12] upon Ibio-number calculation, are specified in footnotes to Table 1 and disregarded in the current study.

The Ibio-number of Centocor's epoetin alfa secondary standard (Ibio_a) was retrospectively calculated by the author from the CZE data published by Centocor [13] according to Formula 1, using the isoform bioactivities communicated by Amgen for epoetin alfa [14]. The Ibio-numbers of Centocor's formulated epoetin alfa 40k (Ibio_a_40k) respectively $2 \mathrm{k}$ (Ibio_a_2k) drug product samples were again based on the CZE data published by Centocor [13] and calculated alike.

The mean Ibio-number of Roche's 40 epoetin beta training batches (Ibio_B_40tr) was calculated from the mean CZE data published by Roche [3] according to Formula 1, using the isoform bioactivities communicated by Roche for epoetin beta [15]. Thus, the average values of isoforms 1-8 of these 40 epoetin beta training batches were regarded as the isoform values of an artificial epoetin beta concentrated solution pool material.

Note: Compared with the Roche paper [3], the isoforms are numbered in reverse order in this paper, which uses the order of the Ph. Eur. monograph (Figure 1) [5].

The mean Ibio-number of Roche's 17 epoetin beta validation batches (Ibio_ß_17val) was calculated via Ibio_B_40tr (133.8 IU/ $\mu \mathrm{g})$,

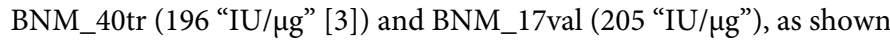
in Formula 2.

\section{Ibio_ß_17val=BNM_17val/BNM_40tr * Ibio_B_40tr}

(Formula 2)

The Ibio-numbers of the two hypothetical epoetin alfa/beta 1:1 mixtures (Ibio_ $\alpha / \beta \_40 \operatorname{tr}$ and Ibio_ $\alpha / \beta \_17 \mathrm{val}$ ) were calculated as the arithmetic mean of Ibio_ $\alpha$ and Ibio_B_40tr respectively Ibio_ $\alpha$ and Ibio_ß_17val (Formula 3).

Ibio_ $\alpha / \beta=($ Ibio_$\alpha+$ Ibio_ß $) / 2$

(Formula 3) 
Citation: Hermentin P (2017) Ibio-Number Assay: A Physicochemical Assay that Predicts the Bioactivity of Erythropoietin with High Precision and Accuracy and May Replace the Mouse Bioassay in the Quality Control of EPO Batch Release. Pharm Anal Acta 8: 533. doi: 10.4172/21532435.1000533

Page 3 of 9

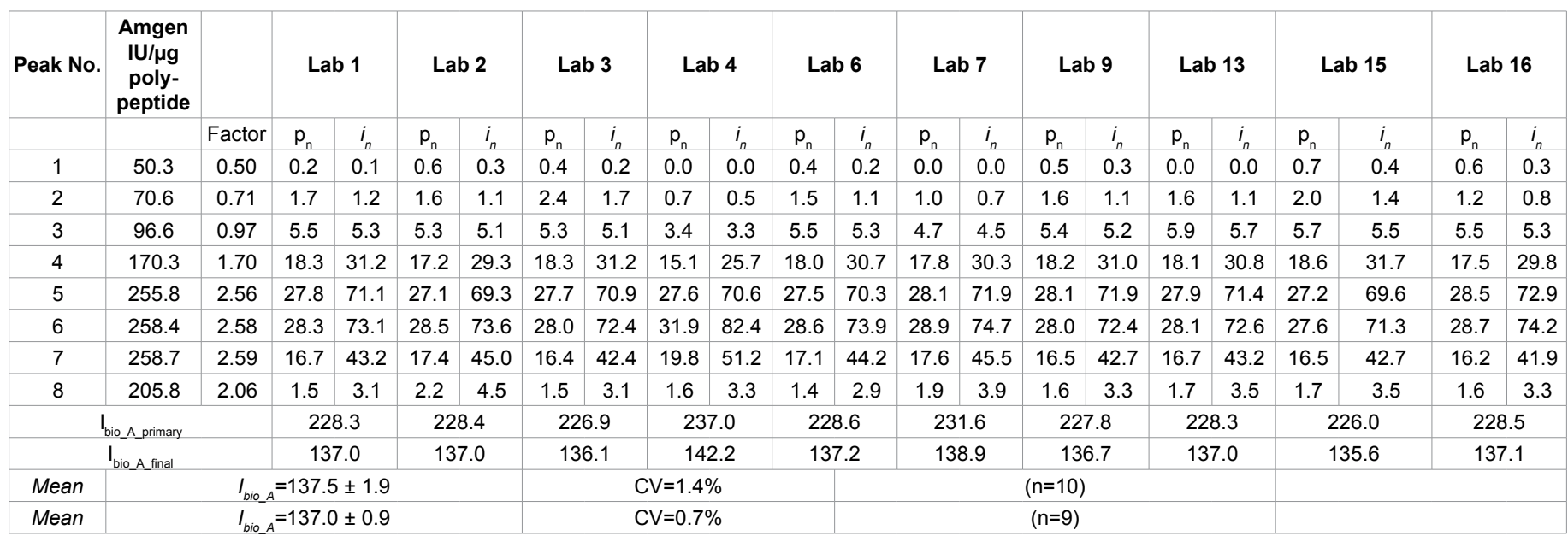

Table 1a: cBRP3 $I$-number calculation and inter-laboratory precision. Basis: Behr-Gross et al. [12]: Table 3d-Isoform distribution (in \%) of cBRP3 uncorrected for migration time. Using isoform bioactivities published by Strickland TW [14]. Note: Lab 4 proved to be an outlier according to Grubbs ( $\alpha=0.01)$ and according to Dixon ( $\alpha=0.02$ ) and was therefore disregarded.

\begin{tabular}{|c|c|c|c|c|c|c|c|c|c|c|c|c|c|c|c|c|c|c|c|c|c|c|}
\hline \multirow{2}{*}{$\begin{array}{c}\text { Peak } \\
\text { No }\end{array}$} & \multirow{2}{*}{ 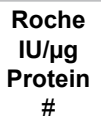 } & & \multicolumn{2}{|c|}{ Lab 1} & \multicolumn{2}{|c|}{ Lab 2} & \multicolumn{2}{|c|}{ Lab 3} & \multicolumn{2}{|c|}{ Lab 4} & \multicolumn{2}{|c|}{ Lab 6} & \multicolumn{2}{|c|}{ Lab 7} & \multicolumn{2}{|c|}{ Lab 9} & \multicolumn{2}{|c|}{ Lab 13} & \multicolumn{2}{|c|}{ Lab 15} & \multicolumn{2}{|c|}{ Lab 16} \\
\hline & & & $p_{n}$ & $i_{n}$ & $p_{n}$ & $i_{n}$ & $p_{n}$ & $i_{n}$ & $p_{n}$ & $i_{n}$ & $p_{n}$ & $i_{n}$ & $p_{n}$ & $i_{n}$ & $p_{n}$ & $i_{n}$ & $p_{n}$ & $i_{n}$ & $p_{n}$ & $i_{n}$ & $p_{n}$ & $i_{n}$ \\
\hline 1 & 19 & 0.19 & 0.2 & 0.0 & 0.6 & 0.1 & 0.4 & 0.1 & 0.0 & 0.0 & 0.4 & 0.1 & 0.0 & 0.0 & 0.5 & 0.1 & 0.0 & 0.0 & 0.7 & 0.1 & 0.6 & 0.1 \\
\hline 2 & 40 & 0.40 & 1.7 & 0.7 & 1.6 & 0.6 & 2.4 & 1.0 & 0.7 & 0.3 & 1.5 & 0.6 & 1.0 & 0.4 & 1.6 & 0.6 & 1.6 & 0.6 & 2.0 & 0.8 & 1.2 & 0.5 \\
\hline 3 & 75 & 0.75 & 5.5 & 4.1 & 5.3 & 4.0 & 5.3 & 4.0 & 3.4 & 2.6 & 5.5 & 4.1 & 4.7 & 3.5 & 5.4 & 4.1 & 5.9 & 4.4 & 5.7 & 4.3 & 5.5 & 4.1 \\
\hline 4 & 150 & 1.50 & 18.3 & 27.5 & 17.2 & 25.8 & 18.3 & 27.5 & 15.1 & 22.7 & 18.0 & 27.0 & 17.8 & 26.7 & 18.2 & 27.3 & 18.1 & 27.2 & 18.6 & 27.9 & 17.5 & 26.3 \\
\hline 5 & 200 & 2.00 & 27.8 & 55.6 & 27.1 & 54.2 & 27.7 & 55.4 & 27.6 & 55.2 & 27.5 & 55.0 & 28.1 & 56.2 & 28.1 & 56.2 & 27.9 & 55.8 & 27.2 & 54.4 & 28.5 & 57.0 \\
\hline 6 & 280 & 2.80 & 28.3 & 79.2 & 28.5 & 79.8 & 28.0 & 78.4 & 31.9 & 89.3 & 28.6 & 80.1 & 28.9 & 80.9 & 28.0 & 78.4 & 28.1 & 78.7 & 27.6 & 77.3 & 28.7 & 80.4 \\
\hline 7 & 400 & 4.00 & 16.7 & 66.8 & 17.4 & 69.6 & 16.4 & 65.6 & 19.8 & 79.2 & 17.1 & 68.4 & 17.6 & 70.4 & 16.5 & 66.0 & 16.7 & 66.8 & 16.5 & 66.0 & 16.2 & 64.8 \\
\hline 8 & 205.8 & 2.06 & 1.5 & 3.1 & 2.2 & 4.5 & 1.5 & 3.1 & 1.6 & 3.3 & 1.4 & 2.9 & 1.9 & 3.9 & 1.6 & 3.3 & 1.7 & 3.5 & 1.7 & 3.5 & 1.6 & 3.3 \\
\hline \multicolumn{3}{|c|}{$\mathrm{I}_{\text {bio R primary }}$} & \multicolumn{2}{|c|}{237.0} & \multicolumn{2}{|c|}{238.7} & \multicolumn{2}{|c|}{234.9} & \multicolumn{2}{|c|}{252.5} & \multicolumn{2}{|c|}{238.2} & \multicolumn{2}{|c|}{242.1} & \multicolumn{2}{|c|}{236.0} & \multicolumn{2}{|c|}{237.0} & \multicolumn{2}{|c|}{234.3} & \multicolumn{2}{|c|}{236.4} \\
\hline \multicolumn{3}{|c|}{$I_{\text {bio_R_final }}$} & \multicolumn{2}{|c|}{142.2} & \multicolumn{2}{|c|}{143.2} & \multicolumn{2}{|c|}{141.0} & \multicolumn{2}{|c|}{151.5} & \multicolumn{2}{|c|}{142.9} & \multicolumn{2}{|c|}{145.2} & \multicolumn{2}{|c|}{141.6} & & 2.2 & & 0.6 & & 1.9 \\
\hline Mean & & $R=143$ & \pm 3.2 & & & & $C V=2.2$ & $\% \pm 1$ & & & & & & & & & & & & & & \\
\hline Mean & $I_{\text {bio_R } R}=1$ & & & & & & $C V=$ & $1.0 \%$ & & & & & & & & & & & & & & \\
\hline
\end{tabular}

Table 1b: Using isoform bioactivities published by Burg J [15]. \#Specific activity of isoform peak no. 8 as published by Strickland TW [14] as the specific activity of this isoform of Burg J [15] was not available. Note: Lab 4 proved to be an outlier according to Grubbs ( $\alpha=0.01)$ and according to Dixon ( $\alpha=0.005)$ and was therefore disregarded.

\begin{tabular}{|c|c|c|c|c|c|c|c|c|c|c|c|}
\hline & Lab no. & 1 & 2 & 3 & 6 & 7 & 9 & 13 & 15 & 16 & \\
\hline \multirow[t]{3}{*}{ cBRP3 } & Amgen & 137.0 & 137.0 & 136.1 & 137.2 & 138.9 & 136.7 & 137.0 & 135.6 & 137.1 & cp. Table 1a \\
\hline & Roche & 142.2 & 143.2 & 141.0 & 142.9 & 145.2 & 141.6 & 142.2 & 140.6 & 141.9 & cp. Table 1b \\
\hline & Mean & 139.6 & 140.1 & 138.6 & 140.0 & 142.1 & 139.1 & 139.6 & 138.1 & 139.5 & \\
\hline \multicolumn{2}{|c|}{ Mean of means } & \multicolumn{3}{|c|}{$139.6 \pm 1.1$} & \multicolumn{2}{|c|}{$C V=0.8 \%$} & \multicolumn{5}{|c|}{ (n=9 labs) } \\
\hline
\end{tabular}

Table 1c: Mean of means

Note: Lab 4 (not shown) proved to be an outlier according to Grubbs $(\alpha=0.01$ ) and according to Dixon ( $\alpha=0.01)$ and was therefore disregarded. Accuracy versus the stated bioactivity of BRP3 $(141.1 \mathrm{IU} / \mu \mathrm{gg})=98.9 \%$ (difference $=-1.1 \%$ ).

\section{Results}

\section{Inter-laboratory precision}

A reference electropherogram is shown in Figure 1.

The Ibio-number calculation for EPO cBRP3 from the CZE data published in the 2007 study [12] provided Ibio_cBRP3=139.6 IU/ $\mu \mathrm{g} \pm 1.1 \mathrm{IU} / \mu \mathrm{g}$, corresponding with an inter-laboratory precision of $\mathrm{CV}=0.8 \%$ ( $\mathrm{n}=9$ labs) and an accuracy against the stated bioactivity of EPO BRP3 (141.1 IU/ $\mu \mathrm{g}=100 \%)$ of $98.9 \%$ (difference=-1.1\%) (Table 1).

\section{Retrospective analysis of epoetin alfa drug substance and drug product samples}

Centocor's epoetin alfa secondary standard [13] provided Ibio_ $\alpha=142.3 \mathrm{IU} / \mu \mathrm{g} \pm 0.1 \mathrm{IU} / \mu \mathrm{g}(\mathrm{CV}=0.1 \% ; \mathrm{n}=4 \mathrm{CZE}$ runs) (Table 2) These results were confirmed in Centocor's validation runs, providing Ibio_a_day1_day $2=142.3 \mathrm{IU} / \mu \mathrm{g} \pm 0.4 \mathrm{IU} / \mu \mathrm{g}(\mathrm{CV}=0.3 \% ; \mathrm{n}=4$ runs on 2 different days) (Table 3).

The Ibio-number assay applied to Centocor's formulated 40,000 $\mathrm{IU} / \mathrm{mL}(40 \mathrm{k})$ epoetin alfa drug product sample after polysorbate 80 removal provided Ibio_a_40k_day1_day $2=139.8 \mathrm{IU} / \mu \mathrm{g} \pm 0.2 \mathrm{IU} /$ $\mu \mathrm{g}(\mathrm{CV}=0.1 \% ; \mathrm{n}=6$ runs on 2 different days $)$ and an accuracy versus Centocor's secondary standard of $98.3 \%$ (difference $=-1.7 \%$ ) (Table 4).

The Ibio-number assay applied to Centocor's formulated 2,000 IU/ $\mathrm{mL}(2 \mathrm{k})$ epoetin alfa drug product sample after polysorbate 80 removal provided Ibio_a_2k_day1_day2=138.1 IU/ $\mu \mathrm{g} \pm 0.2 \mathrm{IU} / \mu \mathrm{g}(\mathrm{CV}=0.1 \%$; $\mathrm{n}=6$ runs on 2 different days) and an accuracy versus Centocor's secondary standard of $97.1 \%$ (difference $=-2.9 \%$ ) (Table 5 ). The change 
Citation: Hermentin P (2017) Ibio-Number Assay: A Physicochemical Assay that Predicts the Bioactivity of Erythropoietin with High Precision and Accuracy and May Replace the Mouse Bioassay in the Quality Control of EPO Batch Release. Pharm Anal Acta 8: 533. doi: 10.4172/21532435.1000533

Page 4 of 9

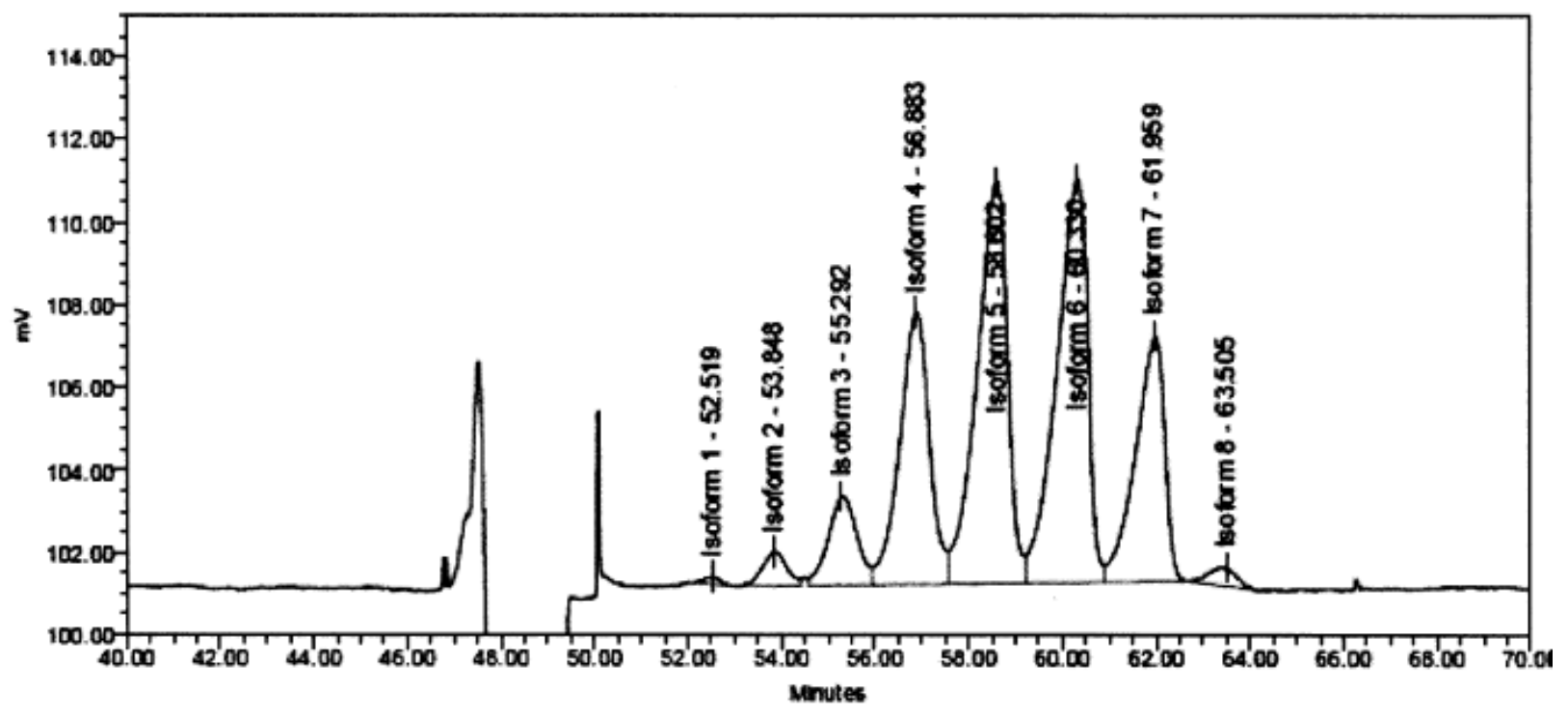

Figure 1: CZE electropherogram of EPO cBRP3, taken from the 2007 study [12]

\begin{tabular}{|c|c|c|c|c|c|c|c|c|c|c|}
\hline \multirow[t]{2}{*}{ Peak No. } & \multirow[t]{2}{*}{$\begin{array}{c}\text { Amgen } \\
\text { IU/Ng } \\
\text { polypeptide }\end{array}$} & \multirow[b]{2}{*}{ Factor } & \multicolumn{2}{|c|}{ Run 1} & \multicolumn{2}{|c|}{ Run 2} & \multicolumn{2}{|c|}{ Run 3} & \multicolumn{2}{|c|}{ Run 4} \\
\hline & & & $p_{n}$ & $i_{n}$ & $p_{n}$ & $i_{n}$ & $p_{n}$ & $i_{n}$ & $\mathbf{p}_{\mathrm{n}}$ & $i_{n}$ \\
\hline 1 & 50.3 & 0.50 & & & & & & & & \\
\hline 2 & 70.6 & 0.71 & & & & & & & & \\
\hline 3 & 96.6 & 0.97 & 2.1 & 2.0 & 2.2 & 2.1 & 2.2 & 2.1 & 2.1 & 2.0 \\
\hline 4 & 170.3 & 1.70 & 18.9 & 32.2 & 19.0 & 32.4 & 18.7 & 31.8 & 18.9 & 32.2 \\
\hline 5 & 255.8 & 2.56 & 30.5 & 78.0 & 30.6 & 78.3 & 30.3 & 77.5 & 30.4 & 77.8 \\
\hline 6 & 258.4 & 2.58 & 30.2 & 78.0 & 30.2 & 78.0 & 30.2 & 78.0 & 30.2 & 78.0 \\
\hline 7 & 258.7 & 2.59 & 17.2 & 44.5 & 17.0 & 44.0 & 17.3 & 44.8 & 17.2 & 44.5 \\
\hline \multirow[t]{3}{*}{8} & 205.8 & 2.06 & 1.3 & 2.7 & 1.2 & 2.5 & 1.3 & 2.7 & 1.2 & 2.5 \\
\hline & $\mathrm{I}_{\text {bio a primary }}$ & & \multicolumn{2}{|c|}{237.4} & \multicolumn{2}{|c|}{237.2} & \multicolumn{2}{|c|}{236.9} & \multicolumn{2}{|c|}{237.0} \\
\hline & $I_{\text {bio a final }}$ & & \multicolumn{2}{|c|}{142.5} & \multicolumn{2}{|c|}{142.3} & \multicolumn{2}{|c|}{142.2} & \multicolumn{2}{|c|}{142.2} \\
\hline Mean & $I_{\text {bio } \alpha}$ & & 142.3 & \pm & 0.1 & \multicolumn{2}{|c|}{$\mathrm{CV}=$} & $0.1 \%$ & \multicolumn{2}{|c|}{$(n=4)$} \\
\hline
\end{tabular}

Table 2: Epoetin alpha secondary standard, $I_{\text {bio }}$-number calculation. Note: CZE data taken from Table 2 of Zhang et al. [13].

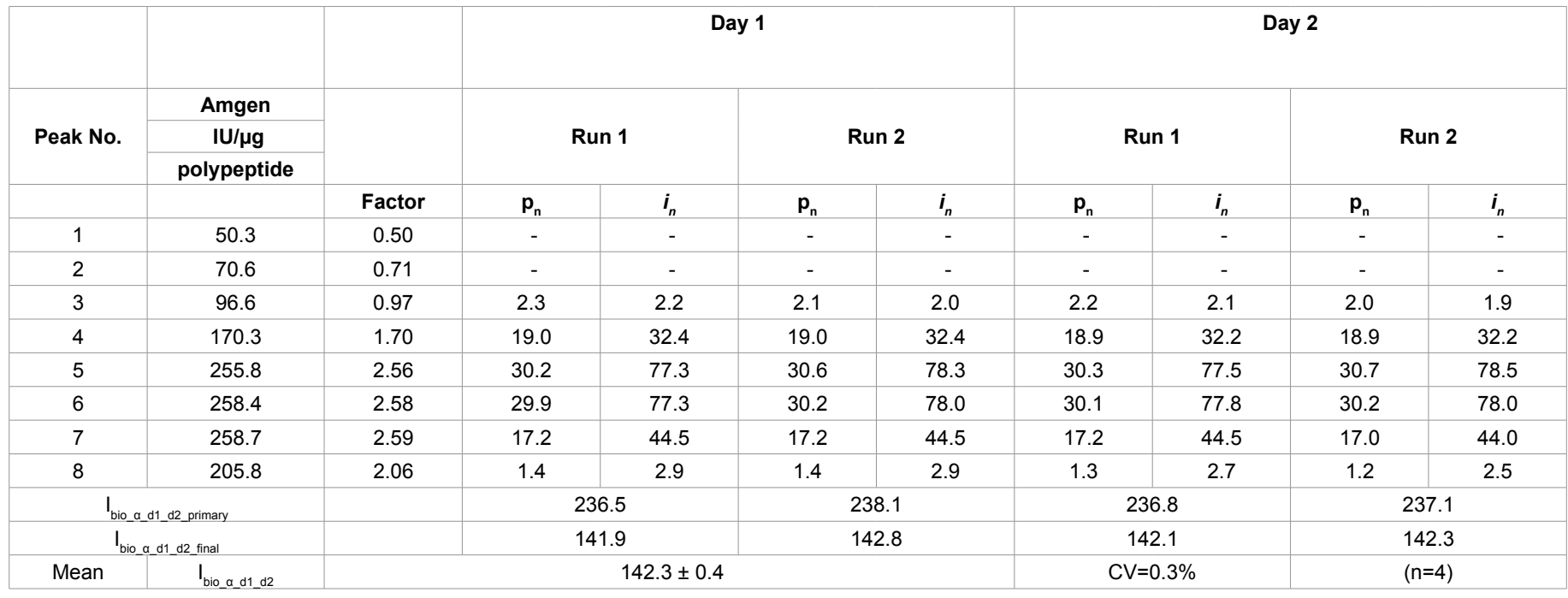

Table 3: Epoetin alfa secondary standard validation runs, Ibio-number calculation Note: CZE data taken from Table 3 of Zhang et al. [13]. 
Citation: Hermentin P (2017) Ibio-Number Assay: A Physicochemical Assay that Predicts the Bioactivity of Erythropoietin with High Precision and Accuracy and May Replace the Mouse Bioassay in the Quality Control of EPO Batch Release. Pharm Anal Acta 8: 533. doi: 10.4172/21532435.1000533

Page 5 of 9

in individual isoform peak areas (pn) after polysorbate 80 removal, compared to the secondary standard, is summarized in Table 6 and commented below.

\section{Retrospective analysis of epoetin beta concentrated solution samples}

Roche's 40 epoetin beta training batches provided a mean Ibio-

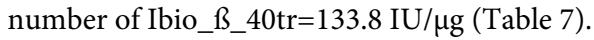

Note: As mentioned in the "Methods", the peak numbering used by Roche [3] was inverse, compared to the peak numbering according to $\mathrm{Ph}$. Eur. [5] respectively the I-number [8] respectively Ibio-number assay $[9,10]$, and the assignment of the isoform bioactivities had to be considered accordingly (Table 7).

The mean Ibio-number of Roche's 17 epoetin beta validation batches was calculated by the author via Formula 2, yielding

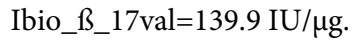

\begin{tabular}{|c|c|c|c|c|c|c|c|c|c|c|c|c|c|c|}
\hline \multirow{3}{*}{ Peak No. } & \multirow{3}{*}{$\begin{array}{c}\text { Amgen } \\
\text { IU/pg } \\
\text { polypeptide }\end{array}$} & \multirow{3}{*}{ Factor } & \multicolumn{6}{|c|}{ Day 1} & \multicolumn{6}{|c|}{ Day 2} \\
\hline & & & \multicolumn{2}{|c|}{ Run 1} & \multicolumn{2}{|c|}{ Run 2} & \multicolumn{2}{|c|}{ Run 3} & \multicolumn{2}{|c|}{ Run 1} & \multicolumn{2}{|c|}{ Run 2} & \multicolumn{2}{|c|}{ Run 3} \\
\hline & & & $p_{n}$ & $i_{n}$ & $p_{n}$ & $i_{n}$ & $p_{n}$ & $i_{n}$ & $p_{n}$ & $i_{n}$ & $p_{n}$ & $i_{n}$ & $\mathbf{p}_{\mathrm{n}}$ & $i_{n}$ \\
\hline 1 & 50.3 & 0.50 & - & - & - & - & - & - & - & - & & & & \\
\hline 2 & 70.6 & 0.71 & - & - & - & - & - & - & - & - & & & & \\
\hline 3 & 96.6 & 0.97 & 3.5 & 3.4 & 3.5 & 3.4 & 3.5 & 3.4 & 3.2 & 3.1 & 3.3 & 3.2 & 3.6 & 3.5 \\
\hline 4 & 170.3 & 1.70 & 20.9 & 35.6 & 20.8 & 35.4 & 20.8 & 35.4 & 21.1 & 35.9 & 20.7 & 35.3 & 20.9 & 35.6 \\
\hline 5 & 255.8 & 2.56 & 29.8 & 76.2 & 29.9 & 76.5 & 29.9 & 76.5 & 30.0 & 76.7 & 30.0 & 76.7 & 29.9 & 76.5 \\
\hline 6 & 258.4 & 2.58 & 28.5 & 73.6 & 28.5 & 73.6 & 28.5 & 73.6 & 28.6 & 73.9 & 28.8 & 74.4 & 28.6 & 73.9 \\
\hline 7 & 258.7 & 2.59 & 15.9 & 41.1 & 16.0 & 41.4 & 16 & 41.4 & 15.8 & 40.9 & 15.9 & 41.1 & 15.8 & 40.9 \\
\hline 8 & 205.8 & 2.06 & 1.30 & 2.7 & 1.3 & 2.7 & 1.3 & 2.7 & 1.3 & 2.7 & 1.3 & 2.7 & 1.2 & 2.5 \\
\hline \multicolumn{2}{|c|}{$I_{\text {bio___40k_primary }}$} & & \multicolumn{2}{|c|}{232.7} & \multicolumn{2}{|c|}{233.0} & \multicolumn{2}{|c|}{233.0} & \multicolumn{2}{|c|}{233.2} & \multicolumn{2}{|c|}{233.4} & \multicolumn{2}{|c|}{232.8} \\
\hline \multicolumn{2}{|c|}{$I_{\text {bi_____ok_final }}$} & & \multicolumn{2}{|c|}{139.6} & \multirow{2}{*}{\multicolumn{2}{|c|}{139.8}} & \multirow{2}{*}{\multicolumn{2}{|c|}{$\begin{array}{c}139.8 \\
C V=0.1 \%\end{array}$}} & \multirow{2}{*}{\multicolumn{2}{|c|}{$\begin{array}{l}139.9 \\
(n=6)\end{array}$}} & \multicolumn{2}{|c|}{140.0} & \multicolumn{2}{|c|}{139.7} \\
\hline Mean & $\mathrm{I}_{\text {bio_a_40k }}$ & & \multicolumn{2}{|c|}{$139.8 \pm 0.2$} & & & & & & & & & & \\
\hline
\end{tabular}

Table 4: $40 \mathrm{k}$ epoetin alfa drug product, Ibio-number calculation. Note: CZE data taken from Table 3 of Zhang et al. [13]. Epoetin alfa secondary standard: Ibio_a=142.3 (cp. Table 3). Accuracy versus secondary standard $=98.3 \%$; difference $=-1.7 \%$

\begin{tabular}{|c|c|c|c|c|c|c|c|c|c|c|c|c|c|c|}
\hline \multirow{3}{*}{ Peak No. } & \multirow{3}{*}{$\begin{array}{c}\text { Amgen } \\
\text { IU/Mg } \\
\text { polypeptide }\end{array}$} & \multirow{3}{*}{ Factor } & \multicolumn{6}{|c|}{ Day 1} & \multicolumn{6}{|c|}{ Day 2} \\
\hline & & & \multicolumn{2}{|c|}{ Run 1} & \multicolumn{2}{|c|}{ Run 2} & \multicolumn{2}{|c|}{ Run 3} & \multicolumn{2}{|c|}{ Run 1} & \multicolumn{2}{|c|}{ Run 2} & \multicolumn{2}{|c|}{ Run 3} \\
\hline & & & $\mathbf{p}_{\mathrm{n}}$ & $i_{n}$ & $\mathbf{p}_{\mathrm{n}}$ & $i_{n}$ & $p_{n}$ & $i_{n}$ & $\mathbf{p}_{\mathrm{n}}$ & $i_{n}$ & $p_{n}$ & $i_{n}$ & $p_{n}$ & $i_{n}$ \\
\hline 1 & 50.3 & 0.50 & - & - & - & - & - & - & - & - & & & & \\
\hline 2 & 70.6 & 0.71 & - & - & - & - & - & - & - & - & & & & \\
\hline 3 & 96.6 & 0.97 & 3.8 & 3.7 & 3.7 & 3.6 & 3.9 & 3.8 & 3.7 & 3.6 & 3.6 & 3.5 & 3.7 & 3.6 \\
\hline 4 & 170.3 & 1.70 & 23.8 & 40.5 & 23.9 & 40.7 & 23.8 & 40.5 & 23.7 & 40.4 & 23.8 & 40.5 & 23.7 & 40.4 \\
\hline 5 & 255.8 & 2.56 & 31.1 & 79.6 & 31.2 & 79.8 & 31.0 & 79.3 & 31.1 & 79.6 & 31.4 & 80.3 & 31.1 & 79.6 \\
\hline 6 & 258.4 & 2.58 & 27.3 & 70.5 & 27.3 & 70.5 & 27.2 & 70.3 & 27.1 & 70.0 & 27.1 & 70.0 & 27.2 & 70.3 \\
\hline 7 & 258.7 & 2.59 & 13.2 & 34.1 & 13.1 & 33.9 & 13.1 & 33.9 & 13.3 & 34.4 & 13.1 & 33.9 & 13.3 & 34.4 \\
\hline 8 & 205.8 & 2.06 & 0.9 & 1.9 & 0.9 & 1.9 & 0.9 & 1.9 & 1.1 & 2.3 & 1.1 & 2.3 & 1.0 & 2.1 \\
\hline \multicolumn{2}{|c|}{$I_{\text {bio_a_2k_primary }}$} & & \multicolumn{2}{|c|}{230.3} & \multicolumn{2}{|c|}{230.4} & \multicolumn{2}{|c|}{229.6} & \multicolumn{2}{|c|}{230.2} & \multicolumn{2}{|c|}{230.5} & \multicolumn{2}{|c|}{230.2} \\
\hline \multicolumn{2}{|c|}{$I_{\text {bio_a_2k_final }}$} & & \multicolumn{2}{|c|}{138.2} & \multirow{2}{*}{\multicolumn{2}{|c|}{138.2}} & \multirow{2}{*}{\multicolumn{2}{|c|}{$\begin{array}{c}137.8 \\
C V=0.1 \%\end{array}$}} & \multicolumn{2}{|c|}{138.1} & \multicolumn{2}{|c|}{138.3} & \multicolumn{2}{|c|}{138.1} \\
\hline Mean & $I_{\text {bio a } 2 \mathrm{k}}$ & \multicolumn{3}{|c|}{$138.1 \pm 0.2$} & & & & & \multicolumn{2}{|c|}{$(n=6)$} & & & & \\
\hline
\end{tabular}

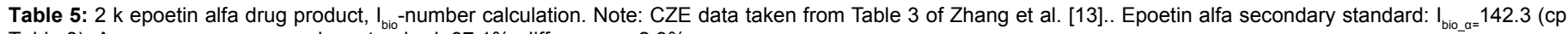
Table 3). Accuracy versus secondary standard $=97.1 \%$; difference $=-2.9 \%$

\begin{tabular}{|c|c|c|c|c|c|}
\hline \multirow{3}{*}{ Peak No. } & \multirow{2}{*}{$\begin{array}{c}\text { Secondary standard } \\
\text { Mean }\end{array}$} & \multicolumn{2}{|c|}{ 40k epoetin } & \multicolumn{2}{|c|}{ 2k epoetin } \\
\hline & & Mean & $P_{n} \%$ & Mean & $P_{n} \%$ \\
\hline & day 1, day 2 & day 1, day 2 & versus standard & day 1 , day 2 & versus standard \\
\hline & $\mathbf{p}_{\mathrm{n}}$ & $\mathbf{p}_{n}$ & & $\mathbf{p}_{\mathrm{n}}$ & \\
\hline 1 & - & - & - & - & - \\
\hline 2 & - & - & - & - & - \\
\hline 3 & 2.15 & 3.43 & 160 & 3.73 & 174 \\
\hline 4 & 18.95 & 20.87 & 110 & 23.78 & 126 \\
\hline 5 & 30.45 & 29.92 & 98 & 31.15 & 102 \\
\hline 6 & 30.10 & 28.58 & 95 & 27.20 & 90 \\
\hline 7 & 17.15 & 15.90 & 93 & 13.18 & 77 \\
\hline 8 & 1.33 & 1.28 & 97 & 0.98 & 74 \\
\hline
\end{tabular}

Table 6: Epoetin alfa, change of individual peak areas of sample versus secondary standard (re-evaluation of the CZE data of Table 3 of Zhang et al. [13]). 
Citation: Hermentin P (2017) Ibio-Number Assay: A Physicochemical Assay that Predicts the Bioactivity of Erythropoietin with High Precision and Accuracy and May Replace the Mouse Bioassay in the Quality Control of EPO Batch Release. Pharm Anal Acta 8: 533. doi: 10.4172/21532435.1000533

Page 6 of 9

\begin{tabular}{|c|c|c|c|c|c|c|}
\hline \multirow{3}{*}{ Peak No. } & Roche & \multirow{3}{*}{ Factor } & \multirow{3}{*}{$\mathbf{p}_{\mathrm{n}}$} & \multirow{3}{*}{$i_{n}$} & \multirow{3}{*}{$\begin{array}{c}\text { Peak designation } \\
\text { Roche }\end{array}$} & \multirow{3}{*}{$\begin{array}{c}\text { Number of sialic } \\
\text { acids }\end{array}$} \\
\hline & $\mathrm{IU} / \mu \mathrm{g}$ & & & & & \\
\hline & protein & & & & & \\
\hline 1 & 19 & 0.19 & 1.60 & 0.3 & IF_8 & 8 \\
\hline 2 & 40 & 0.40 & 4.63 & 1.9 & IF_7 & 9 \\
\hline 3 & 75 & 0.75 & 9.06 & 6.8 & IF_6 & 10 \\
\hline 4 & 150 & 1.50 & 15.25 & 22.9 & IF_5 & 11 \\
\hline 5 & 200 & 2.00 & 24.89 & 49.8 & IF_4 & 12 \\
\hline 6 & 280 & 2.80 & 26.48 & 74.1 & IF_3 & 13 \\
\hline 7 & 400 & 4.00 & 15.77 & 63.1 & IF_2 & 14 \\
\hline 8 & \#) 205.8 & 2.06 & 2.03 & 4.2 & IF_1 & - \\
\hline \multicolumn{3}{|c|}{ I bio_B_40tr_primary } & \multicolumn{2}{|c|}{223.0} & & \\
\hline \multicolumn{3}{|c|}{$\mathrm{I}_{\text {bio_B_ } 40 \mathrm{tr} \text { ffinal }}$} & \multicolumn{2}{|c|}{133.8} & & \\
\hline
\end{tabular}

Table 7: 40 Epoetin beta training batches, $I_{\text {bio }}$-number calculation. Note: CZE data taken from Table 2 of Zimmermann et al. [3]. \#) Specific activity of isoform peak no. 8 as published by Strickland TW [14] as the specific activity of this isoform of Burg J [15] was not available.

\section{Retrospective analysis of an epoetin alfa/epoetin beta 1:1 mixture}

Centocor's epoetin alfa secondary standard and Roche's mean of the 40 epoetin beta training batches, when theoretically mixed 1:1,

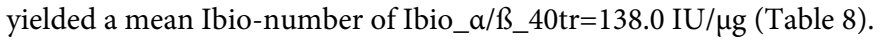

The accuracy of Ibio_ $\alpha / \beta \_40$ tr against Ibio_cBRP3 $(=100 \%)$ and the stated bioactivity of BRP3 (=100\%) were $98.9 \%$ and $97.8 \%$, respectively, revealing differences of $-1.1 \%$ and $-2.2 \%$, respectively (Table 8 ).

Centocor's epoetin alfa secondary standard and Roche's mean of the 17 epoetin beta validation batches, when theoretically mixed 1:1,

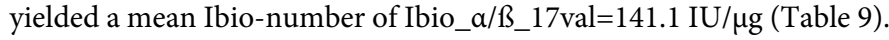

The accuracy of Ibio_a/B_17val against Ibio_cBRP3 $(=100 \%)$ and the stated bioactivity of BRP3 $(=100 \%)$ were $101.1 \%$ and $100.0 \%$, respectively, revealing differences of $+1.1 \%$ and $0.0 \%$, respectively (Table 9 ).

\section{Discussion}

The reduction, refinement or replacement (3Rs) of bioassays is "a common goal of animal welfare societies, health authorities and pharmaceutical manufacturers" [3]. In this regard, efforts to replace the EPO bioassay in normocythaemic and polycythaemic mice has resulted in the I-number assay [8-10] using integers corresponding with the peak number in CZE on the one hand side, respectively the "Ibio"-number assay which was already earlier described $[9,10]$, however not termed as such, using the bioactivity of the isoforms for "Ibio"-number calculation as presented herein, on the other hand side. A third approach has been introduced by Roche to replace the normocythaemic mouse bioassay of epoetin beta batch release [3] that likewise uses the CZE data gained from the epoetin beta samples, however takes only 5 epoetin beta isoforms into consideration instead of taking all 8 isoforms (where applicable) as demanded by Ph. Eur. [5] and the I-number respectively Ibio-number assay [8-10], and instead adds a term that reflects the degree of sialylation. This study from Roche has "shown that the biological activity of erythropoietin can be precisely measured by CZE under the precondition that the variance of its glycosylation does not exceed defined limits" [3]. Hence, the Roche assay has in the meanwhile been accepted by the European authorities for epoetin beta drug substance batch release [11].

The data summarized herein aim at proving that the Ibionumber assay is likewise suitable to calculate the biological activity of erythropoietin and, hence, may likewise be suitable to replace the mouse bioassay of EPO drug substance batch release.

\section{Retrospective analysis of cBRP3}

The data summarized herein may be regarded as a retrospective analysis by the author of CZE data of a collaborative study [12], dealing with EPO cBRP3, with respect to "inter-laboratory precision" (same material, different labs) and "accuracy"-comparison with the stated bioactivity of EPO BRP3 (=100\%) as the "true" bioactivity value.

The fact that the final Ibio-number calculation for EPO cBRP3 was averaged ("mean of means") based on the isoform bioactivities communicated by Amgen [14] as well as by Roche [15] is rationalized by the fact that cBRP3 was in fact a 1:1 mixture of epoetin alfa (from Amgen) and epoetin beta (from Roche) [12]. In this regard, it needs to be emphasized that the isoform bioactivities published by Amgen for epoetin alfa respectively Roche for epoetin beta were expressed in "units/mg erythropoietin polypetide" [14] respectively "IU/mg protein" [15]. Hence, the primary Ibio-numbers calculated therefrom had to be "multiplied by the factor 0.60 polypetide/mg erythropoietin glycoprotein to give specific activities expressed as units/mg erythropoietin glycoprotein" [14] respectively the final Ibio-numbers.

As can be seen from Table 1, the Ibio-number assay, applied to the 2007 collaborative study [12], yielded a bioactivity of Ibio_cBRP3 $=139.6$ $\mathrm{IU} / \mu \mathrm{g}$, with an inter-laboratory precision of $\mathrm{CV}=0.8 \%$ ( $\mathrm{n}=9$ labs; outlier lab 4 excluded-see footnotes to Table 1), and an accuracy (against the stated potency of BRP3) of $98.9 \%$ (difference $=-1.1 \%$ ) (Table 1).

This precise set of data primarily relies on the precision and accuracy of CZE. But exactly for that reason, the precision of the Ibionumber determination of EPO cBRP3 (respectively EPO samples) simultaneously reflects the bioactivity of the samples in CZE in a likewise precise manner, which was likewise apparent from the hypothetical epoetin alfa/beta 1:1 mixtures, which is further discussed below.

The accordance of Ibio_cBRP3 (139.6 IU/ $\mu \mathrm{g})$ with the stated bioactivity of EPO BRP3 (141.1 IU/ $\mu \mathrm{g})$ may be taken as a proof of principle that the Ibio-number of an EPO sample reflects the potency of the sample, which was further supported by the epoetin alfa/beta 1:1 mixtures presented below. 
Citation: Hermentin P (2017) Ibio-Number Assay: A Physicochemical Assay that Predicts the Bioactivity of Erythropoietin with High Precision and Accuracy and May Replace the Mouse Bioassay in the Quality Control of EPO Batch Release. Pharm Anal Acta 8: 533. doi: 10.4172/21532435.1000533

Page 7 of 9

\begin{tabular}{|c|c|c|c|c|c|c|c|}
\hline Material & \multicolumn{2}{|c|}{$\mathrm{I}_{\mathrm{bio}}-$ number $[\mathrm{IU} / \mu \mathrm{g}]$} & \multirow{2}{*}{\begin{tabular}{|c|}
$\begin{array}{c}\text { Data } \\
\text { from }\end{array}$ \\
Table 2 \\
\end{tabular}} & \multirow[b]{2}{*}{-} & \multirow[b]{2}{*}{-} & \multirow[b]{2}{*}{-} & \multirow[b]{2}{*}{-} \\
\hline $\begin{array}{l}\text { Epoetin alfa secondary standard } \\
\text { (Centocor) }\end{array}$ & $\mathrm{I}_{\text {bio_a }}$ & 142.3 & & & & & \\
\hline $\begin{array}{l}\text { Epoetin beta, mean of } 40 \text { 'training } \\
\text { batches' (Roche) }\end{array}$ & $\mathrm{I}_{\text {bi____40tr }}$ & 133.8 & Table 7 & - & - & - & - \\
\hline Epoetin $\alpha / \beta \_40$ tr & \multirow[b]{2}{*}{$\mathrm{I}_{\text {bio_a//3_4otr }}$} & \multirow[b]{2}{*}{138.0} & & $\begin{array}{c}\text { Accuracy } \\
\text { against } \\
I_{\text {bio_cBRP3 }}\end{array}$ & $98.9 \%$ & Difference to $I_{\text {bio_cBRP3 }}$ & $-1.1 \%$ \\
\hline (1:1 mixture) & & & & $\begin{array}{l}\text { Accuracy } \\
\text { against } \\
\text { stated } \\
\text { bioactivity of } \\
\text { BRP3 }\end{array}$ & $97.8 \%$ & $\begin{array}{l}\text { Difference to stated } \\
\text { bioactivity of BRP3 }\end{array}$ & $-2.2 \%$ \\
\hline cBRP3, ref [14] & \multirow{2}{*}{$\mathrm{I}_{\text {bio_cBRP3 }}$} & \multirow{2}{*}{139.6} & \multirow{2}{*}{ Table 1} & \multirow{2}{*}{-} & \multirow{2}{*}{-} & \multirow{2}{*}{-} & \multirow{2}{*}{-} \\
\hline ( $n=9$ labs $)$ & & & & & & & \\
\hline BRP3, stated bioactivity [IU//ng] & & 141.1 & & - & - & - & - \\
\hline
\end{tabular}

Table 8: Epoetin alfa/beta_40tr 1:1 mixture, $\mathrm{I}_{\text {bio }}$-number calculation, accuracy determination.

\begin{tabular}{|c|c|c|c|c|c|c|c|}
\hline Material & \multicolumn{2}{|c|}{$\mathrm{I}_{\mathrm{bio}}-$-number $[\mathrm{IU} / \mu \mathrm{g}]$} & \multirow{2}{*}{\begin{tabular}{|c|}
$\begin{array}{c}\text { Data } \\
\text { from }\end{array}$ \\
Table 2 \\
\end{tabular}} & \multirow[b]{2}{*}{-} & \multirow[b]{2}{*}{-} & \multirow[b]{2}{*}{-} & \multirow[b]{2}{*}{-} \\
\hline $\begin{array}{l}\text { Epoetin alfa secondary standard } \\
\text { (Centocor) }\end{array}$ & $\mathrm{I}_{\text {bio_a }}$ & 142.3 & & & & & \\
\hline $\begin{array}{c}\text { Epoetin beta, mean of } 17 \text { 'validation } \\
\text { batches' (Roche) }\end{array}$ & $\mathrm{I}_{\text {bio_ß__17val }}$ & 139.9 & - & - & - & - & - \\
\hline Epoetin $\alpha / \beta \_17 v a l$ & \multirow[b]{2}{*}{$\mathrm{I}_{\text {bio_a/3_17val }}$} & \multirow[b]{2}{*}{141.1} & \multirow[b]{2}{*}{-} & $\begin{array}{c}\text { Accuracy } \\
\text { against } \\
I_{\text {bio_cBRP3 }}\end{array}$ & $101.1 \%$ & Difference to $\mathrm{I}_{\text {bio_cBRP3 }}$ & $1.1 \%$ \\
\hline (1:1 mixture) & & & & $\begin{array}{l}\text { Accuracy } \\
\text { against } \\
\text { stated } \\
\text { bioactivity } \\
\text { of BRP3 }\end{array}$ & $100.0 \%$ & $\begin{array}{l}\text { Difference to stated } \\
\text { bioactivity }\end{array}$ & $0.0 \%$ \\
\hline cBRP3 [14] & \multirow{2}{*}{$\mathrm{I}_{\text {bio_cBRP3 }}$} & \multirow{2}{*}{139.6} & \multirow{2}{*}{ Table 1} & \multirow{2}{*}{-} & \multirow{2}{*}{-} & \multirow{2}{*}{-} & \multirow{2}{*}{-} \\
\hline ( $n=9$ labs) & & & & & & & \\
\hline BRP3, stated bioactivity [IU/ $/ \mu \mathrm{g}]$ & - & 141.1 & - & - & - & - & - \\
\hline
\end{tabular}

Table 9: Epoetin alfa/beta_17val 1:1 mixture, $\mathrm{I}_{\text {bio }}$-number calculation, accuracy determination.

\section{Retrospective analysis of epoetin alfa drug substance and drug product samples}

An attempt to compare the calculated bioactivity of Centocor's

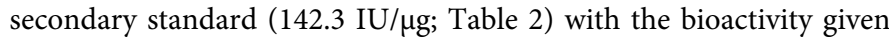
by Centocor [13] was deliberately omitted as Centocor's epoetin alfa secondary standard has been calibrated against EPO BRP1, the stated bioactivity of which has been questioned by the author elsewhere [18].

The Ibio-number of Centocor's epoetin alfa secondary standard was retrospectively calculated by the author from the CZE data of the four validation runs published in the Centocor study [13], which yielded a mean bioactivity of Ibio_ $\alpha=142.3 \mathrm{IU} / \mu \mathrm{g} \pm 0.1 \mathrm{IU} / \mu \mathrm{g}$ (Table 2 ).

Two sets of experiments were performed by Centocor [13] on two different days, with three CZE runs per sample and per day, each, in order to prove in a kind of limited validation (determination of "repeatability" and "intermediate precision") that their formulated epoetin alfa drug product ( $40 \mathrm{k}$ and $2 \mathrm{k}$ ) samples could be satisfactorily measured in CZE after polysorbate 80 removal and desalting. No "accuracy" determination was performed by Centocor with their epoetin alfa drug product samples after polysorbate 80 removal, and this "accuracy" determination was evaluated and supplemented herein, using the Ibio-number assay.

The Ibio-number assay applied to Centocor's epoetin alfa secondary standard, which was run in duplicate injection, each day, provided the practically identical mean bioactivity of Ibio_a_day1_day2=142.3 IU/ $\mu \mathrm{g}$ as before, however with a slightly inferior intermediate precision, $\mathrm{CV}=0.3 \%$ ( $\mathrm{n}=4$ runs on two different days) (Table 3 ).

The formulated $40 \mathrm{k}$ epoetin alfa drug product sample after polysorbate 80 removal provided a bioactivity of Ibio_a_40 k_day1_ day $2=139.8 \mathrm{IU} / \mu \mathrm{g} \pm 0.2 \mathrm{IU} / \mu \mathrm{g}$ (Table 4$)(\mathrm{CV}=0.1 \% ; \mathrm{n}=6$ runs on two different days) and an accuracy versus Centocor's secondary standard of $98.3 \%$, respectively a difference of $-1.7 \%$, which rendered the $40 \mathrm{k}$ sample "comparable" to the secondary standard ("comparable" at differences of 1.5-3.0 IU/ $\mu \mathrm{g}$ [18]).

The formulated $2 \mathrm{k}$ epoetin alfa drug product sample after polysorbate 80 removal yielded a bioactivity of Ibio_a_2k $=138.1$ $\mathrm{IU} / \mu \mathrm{g} \pm 0.2 \mathrm{IU} / \mu \mathrm{g}$ (Table 5) $(\mathrm{CV}=0.1 \% ; \mathrm{n}=6$ runs on two different days) and an accuracy versus Centocor's secondary standard of 97.1\% (difference $=-2.9 \%$ ), which rendered the $2 \mathrm{k}$ sample borderline "comparable" to the secondary standard.

The slight decrease in the Ibio-number observed for the $40 \mathrm{k}$ sample (-1.7\% Table 4$)$ and the $2 \mathrm{k}$ sample (-2.9\%, Table 5), was caused by a change in the isoform composition observed after polysorbate 80 removal, which was, however, not obvious just like that from the Centocor paper [13], which focused on the precision of the isoform peaks of the $40 \mathrm{k}$ and the $2 \mathrm{k}$ samples after polysorbate 80 removal. ("Precision ranged from $0.0 \%$ to $6.5 \%$. The relative area of each isoform for $2 \mathrm{k} \mathrm{IU} / \mathrm{mL}$ F-EPO and $40 \mathrm{k} \mathrm{IU} / \mathrm{mL}$ F-EPO differed from the epoetin alfa secondary standard by $0.7 \%$ or less, and were considered to be equivalent to that of the epoetin alfa standard" [13]). Centocor did 
Citation: Hermentin P (2017) Ibio-Number Assay: A Physicochemical Assay that Predicts the Bioactivity of Erythropoietin with High Precision and Accuracy and May Replace the Mouse Bioassay in the Quality Control of EPO Batch Release. Pharm Anal Acta 8: 533. doi: 10.4172/21532435.1000533

Page 8 of 9

not comment on the change of individual peak areas of sample versus secondary standard. Thus, the isoform percentage shares of isoforms 3 and 4 were significantly higher, whereas those of isoforms 6 and 7 were lower after polysorbate 80 removals, compared with the isoform percentage shares of the secondary standard, which was particularly apparent from the $2 \mathrm{k}$ drug product sample (Table 6).

Thus, isoform 3 increased from $2.15 \%$ (secondary standard: mean of $n=4$ runs on two different days) to $3.73 \%$ ( $2 \mathrm{k}$ drug product sample, $\mathrm{n}=6$ runs on two different days), equivalent to a 1.7 -fold increase. And isoform 7 decreased from $17.15 \%$ (secondary standard: mean of $n=4$ runs on two different days) to $13.18 \%$ ( $2 \mathrm{k}$ drug product sample, $\mathrm{n}=6$ runs on two different days), equivalent to a decrease of $23 \%$ (Table 6). However, the specification of the EPO monograph 1316 [5] (isoform 3: $1-20 \%$, isoform $7: 5-25 \%$ ) was in each case still fully met.

Noteworthy, epoetin alfa samples are devoid of isoforms 1 and 2, as a result of the epoetin alfa downstream processing, which is well known [19,20]. Thus, Amgen EPO [19] as well as Eprex from JanssenOrtho and the biosimilar Binocrit from Sandoz, which also are epoetin alfa, are devoid of isoforms 1 and 2, and the same holds true for the biosimilar Retacrit from Norbitec/Hospira (epoetin zeta) [20]. This lack in the Centocor sample of isoforms 1 and 2 (which have the lowest isoform bioactivities compared to the other isoforms of the epoetin alfa sample [14] respectively epoetin beta sample [15] may explain whyapart from differences in the carbohydrate moiety-the Ibio-number of the epoetin alfa sample exhibited a higher Ibio-number (Ibio_ $\alpha=142.3$ $\mathrm{IU} / \mu \mathrm{g}$ ) (Table 2) than the artificial mixture of the 40 epoetin beta

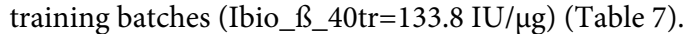

An attempt to compare the calculated bioactivities with the bioactivities given by Centocor ( $2 \mathrm{k}$ sample: $16.8 \mu \mathrm{g} / \mathrm{mL} ; 40 \mathrm{k}$ sample: $336.0 \mu \mathrm{g} / \mathrm{mL}$ ) [13] was deliberately omitted as Centocor's epoetin alfa secondary standard (from which the $2 \mathrm{k}$ and $40 \mathrm{k}$ samples were prepared) has been calibrated against EPO BRP1, the stated bioactivity of which has been questioned by the author [18]

\section{Epoetin beta concentrated solution samples}

Roche communicated the mean BNM of the 40 epoetin beta training batches (BNM40tr=196 IU/ $\mu \mathrm{g}$ ) [3], whereas the mean BNM of the 17 validation batches $(\mathrm{BNM} 17 \mathrm{val}=205.0 \mathrm{IU} / \mu \mathrm{g})$ had to be calculated by the author from the individual BNMs of the 17 validation batches given by Roche [3]. And whereas the mean percentages of Roche's epoetin beta isoforms separated in CZE were again provided for the 40 training batches [3], thus allowing to calculate the corresponding mean bioactivity via the Ibio-number assay (Table 7), the CZE data of the 17 epoetin beta validation batches were not communicated by Roche, and therefore the Ibio_B_17val-number was calculated by the author according to Formula 2, yielding $139.9 \mathrm{IU} / \mu \mathrm{g}$

This calculation may be questioned as it was unclear whether the 17 validation batches had been measured by Roche against EPO BRP2 or EPO BRP3 [3]. This is important to know insofar as it has been suggested by the author that the potency of BRP2 has been stated about $10 \%$ too low, whereas the potency of BRP3 was without any doubt [18].

Noteworthy, the mean biological activity determined in normocythaemic mice (BNM) of Roche's 40 epoetin beta training batches was communicated as $196 \mathrm{IU} / \mu \mathrm{g}$ [3]. Although not explicitly mentioned by Roche, this value must be read as 196 "IU/ $\mu$ g protein", concluded by the author from reference [15], and must therefore be multiplied by 0.60 in order to get "IU/ $\mu$ g EPO" glycoprotein, reflecting "the protein content of the erythropoietin glycoprotein (about $60 \%$ by weight)" [14] respectively 59\% [3].
Quite confusing for the author was, however, the fact that the Ibio_B_40tr $=133.8 \mathrm{IU} / \mu \mathrm{g}$ value $=100 \%$ (Table 7 ) differed from the mean

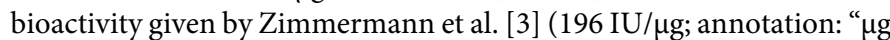
protein") respectively 117.6 "IU/ $\mu \mathrm{g}$ active substance" (as calculated by the author, considering the $\sim 60 \%$ protein share of the EPO molecule) by $-12.1 \%$. Such high difference could be attributable to the fact that batches with borderline isoform distribution had been included in these 40 epoetin beta training batches in order to completely cover the target range of specific activities given by Ph. Eur. ("potency of not less than $100000 \mathrm{IU} / \mathrm{mg}$ of active substance"; "the estimated potency is not less than 80 per cent and not more than $125 \%$ of the stated potency" [5]). Indeed, the potency ranges of their epoetin beta training batches were reported by Roche [3] in the range of 138 "IU/ $\mu \mathrm{g}$ " (annotation: protein) to 229 "IU/ $\mu \mathrm{g}$ " (annotation: protein), with a mean biological activity of 196 "IU/ $\mu$ g" (annotation: protein), which (after multiplication by 0.60

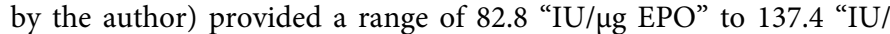

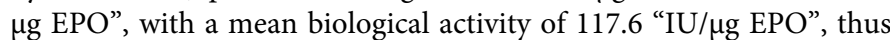
covering the $\mathrm{Ph}$. Eur. target range, as desired. Such high difference of $-12.1 \%$ between the Ibio-number calculated by the author $(=100 \%)$ and the mean BNM given by Roche may, however, and more likely, be attributable to the fact that the 40 epoetin beta training batches have been calibrated against EPO BRP2 [3], the stated potency of which has been stated about $10 \%$ too low [18]

In their first German paper [3] (part 1), Zimmermann et al. stated that they didn't succeed in calculating the bioactivity of their EPO samples via simple addition of the isoforms, referring to an internal report. This initial failure may have been attributable to calibration against BRP1 and/or BRP2, the stated potencies of which have been questioned [18]. In their main paper, however, Zimmermann et al. [3] admitted that "in spite of different published findings [ref], no influence of the N-glycan structures, namely antennarity and repeats, could be seen" [3], which has shown "that the biological activity of erythropoietin can be precisely measured by CZE under the precondition that the variance of its glycosylation does not exceed defined limits" [3], thus confirming the basis of the Ibio-number approach $[9,10]$. Perhaps this later statement is based on calibration against EPO BRP3, the stated potency of which was without any doubt [18].

\section{Retrospective analysis of an epoetin alfa/epoetin beta 1:1 mixture}

Considering the inconsistencies revealed for Centocor's epoetin alfa secondary standard (due to its calibration against EPO BRP1) respectively for Roche's mean of the 40 epoetin beta training batches (due to their calibration against EPO BRP2), it is amazing that the bioactivity of the epoetin alfa/beta_40tr 1:1 mixture calculated via the Ibio-number assay $(138.0 \mathrm{IU} / \mu \mathrm{g})$ fitted pretty well with Ibio_cBRP3=139.6 IU/ $\mu \mathrm{g}$, exhibiting a difference of $-1.1 \%$ (Table 8), which rendered the bioactivity of these two samples analytically "indistinguishable" ("indistinguishable" at differences of $<1.5 \%$ [18]). Ibio_ $\alpha / \beta \_40 \operatorname{tr}=138.0 \mathrm{IU} / \mu \mathrm{g}$ fitted likewise well with the stated

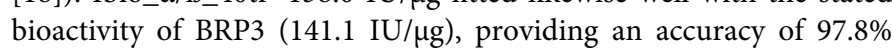
respectively a difference of $-2.2 \%$ (Table 8 ), which rendered the two bioactivities "comparable" ("comparable at differences of 1.5-3.0\% [18]).

Likewise, the bioactivity of the epoetin alfa/beta_17val 1:1 mixture calculated by the author $(141.1 \mathrm{IU} / \mu \mathrm{g})$ was in accordance with Ibio cBRP3 $=139.6 \mathrm{IU} / \mu \mathrm{g}$ (accuracy $=101.1 \%$; difference $=+1.1 \%$ (Table 9), rendering these two samples analytically "indistinguishable" (difference $<1.5 \%$ [18]). And the calculated Ibio_ $\alpha / \beta_{1} \_17$ val value fitted incredibly well with the sated bioactivity of BRP3, providing an accuracy of $100.0 \%$ (Table 9) 
Citation: Hermentin P (2017) Ibio-Number Assay: A Physicochemical Assay that Predicts the Bioactivity of Erythropoietin with High Precision and Accuracy and May Replace the Mouse Bioassay in the Quality Control of EPO Batch Release. Pharm Anal Acta 8: 533. doi: 10.4172/21532435.1000533

Page 9 of 9

Of course, one may object that the isoform bioactivities of epoetin alfa [14] and epoetin beta [15] have been determined many years ago and that these determinations have been highly inaccurate due to the limited number of replicate determinations and the inaccuracy of the mouse bioassay $(\mathrm{CV} \approx 25 \%[1,2], \approx 20 \%[3])$. But it appears that the uncertainties associated with the bioassay of the isolated isoforms have been overcome in the final Ibio_A respectively Ibio_R respectively "mean of means" calculation (Table 1, Tables 8 and 9), as the calculated values fitted so well with the expected potency values.

On the other hand side, the bioactivity of $\mathrm{BNM}=196$ "IU/ $\mu \mathrm{g}$ " (annotation: protein) communicated by Roche for its mean BNM of the 40 epoetin beta training batches [3] respectively 117.6 "IU/ug EPO" as calculated by the author would not fit to the hypothetical epoetin alfa/beta 1:1 mixture as this epoetin beta potency would demand for the potency of the epoetin alfa sample (to be added to yield a 50:50 blend) a value of $164.6 \mathrm{IU} / \mu \mathrm{g}$ (calculated according to Formula 3 ). This value appears to be close to (or even above) the upper potency limit given by Ph. Eur. ("a potency of not less than $100000 \mathrm{IU} / \mathrm{mg}$ of active substance" with an "estimated potency of ... not more than 125 per cent of the stated potency" [5]) and is therefore unlikely.

In contrast, the potencies calculated for the two hypothetical epoetin alfa/beta 1:1 mixtures via the Ibio-number assay (138.0 IU/ $\mu \mathrm{g}$, cp. Table 8; $141.1 \mathrm{IU} / \mu \mathrm{g}$, cp. Table 9) were in accordance with the stated bioactivity of EPO BRP3 (141.1. IU/ $\mu \mathrm{g}$ ), exhibiting differences of $-2.2 \%$ (Table 8 ) and $0.0 \%$ (Table 9), respectively, judged by the author as analytically "comparable" respectively "indistinguishable" [18].

Thus, the accordance of the two hypothetical epoetin alfa/beta 1:1 mixtures with both the calculated and the stated bioactivity of EPO BRP3 was extraordinary, which parallels and confirms the conclusion already drawn above with EPO cBRP3 that Ibio-number determination of an EPO sample via CZE provides its potency in a precise and accurate manner.

\section{Conclusion}

The data presented in this retrospective analysis by the author of the CZE data of various published EPO samples has shown that the Ibio-number assay is suitable to assess the bioactivity of EPO medicinal products with high precision and accuracy, which should be confirmed in further studies comprising in particular samples from EPO batch release.

In combination with other physicochemical assays such as RPHPLC, peptide mapping and HPAEC, the Ibio-number assay offers an opportunity to replace the highly contested (consumption of animals) and highly inaccurate mouse bioassay of EPO batch release.

A prospective collaborative validation study could show whether the assay is suitable to replace the mouse bioassay in the quality control of erythropoietin, as suggested.

\section{References}

1. Storring PL, Gaines Das RE (1992) The international standard for recombinan DNA-derived erythropoietin: collaborative study of four recombinant DNAderived erythropoietins and two highly purified human urinary erythropoietins. J Endocrinology 134: 459-484

2. Hermentin P, Witzel R, Schwick-Wagner P, Blumrich M (2002) N-glycan charge assay - an alternative for potency assays of therapeutic glycoproteins?. Dev Biol 111: 89-97.

3. Zimmermann H, Gerhard D, Hothorn LA, Dingermann T (2011) An Alternative to Animal Testing in the Quality Control of Erythropoietin. Pharmeur Bio Sci Notes 1: 66-80.

4. Bristow A (2008) Possible approaches for replacement of erythropoietin in vivo potency assay. In: EDQM, editors. Alternatives to animal testing: New approaches in the development and control of biologicals. Proceedings of the International Symposium: Dubrovnik, Croatia.

5. (2014) Erythropoietin concentrated solution, monograph 1316, Ph. Eur. 8.0 Strasbourg, France: Council of Europe, 2162-2166.

6. Hermentin P, Witzel R, Kanzy EJ, Diderrich G, Hoffmann D, et al. (1996) The hypothetical $\mathrm{N}$-glycan charge: a number that characterizes protein glycosylation. Glycobiology 6: 217-230.

7. Hermentin P, Witzel R (1999) The hypothetical N-glycan charge: a number to characterize protein N-glycosylation. Pharm Pharmacol Commun 5: 33-43.

8. Hermentin P (2006) Isoform number I - a new tool to evaluate the quality of erythropoietin. Pharmeuropa Scientific Notes 1: 37-40.

9. Hermentin P (2009) Method for characterizing the glycosylation of sialoglycoproteins via an isoform number I. EP 1943524 B1.

10. Hermentin P (2012) Method for characterisation of the quality of sialoglycoproteins via an isoform number I. US 8,150,630 B2.

11. Schnueriger F (2013) CMC Strategy Forum Europe, Hilton Old Town, Prague Czech Republic - see slide 20: "Approach accepted for DS, in vivo assay still in use for DP".

12. Behr-Gross ME, Daas A, Burns C, Bristow AF (2007) Collaborative study for the establishment of erythropoietin BRP batch 3. Pharmeuropa Bio 1: 49-66.

13. Zhang J, Chakraborty U, Villalobos AP, Brown JM, Foley JP (2009) Optimization and qualification of capillary zone electrophoresis method for glycoprotein isoform distribution of erythropoietin for quality control laboratory. J Pharm Biomed Anal 50: 538-543.

14. Strickland TW, Byrne TE, Elliott SG (1996) Erythropoietin isoforms, EP 0428 267 B1.

15. Burg J, Sellinger KH, Haselbeck A, Koll H (2014) Erythropoietin with high specific activity. EP $1037921 \mathrm{~B} 1$

16. Grubbs F (1969) Procedures for detecting outlying observations in samples. Technometrics 11: 1-21.

17. Dixon WJ (1950) Analysis of extreme values. Ann Math Stat 21: 488-506.

18. Hermentin $P$ (2017) Evidence that the potencies of the biological reference preparations batch 1 and batch 2 of erythropoietin have been stated $\sim 5 \%$ too low respectively $\sim 10 \%$ too low (submitted).

19. Park SS, Park J, Ko J, Chen L, Meriage D, et al. (2009) Biochemical assessment of erythropoietin products from Asia versus US Epoetin alfa manufactured by Amgen. J Pharm Sci 98: 1688-1699.

20. Brinks V, Hawe A, Basmeleh AH, Joachin-Rodriguez L, Haselberg R, et al. (2011) Quality of original and biosimilar epoetin products. Pharm Res 28: 386-393. 